\title{
Impacto económico y social de la asistencia personal a través de la metodología del Retorno Social de la Inversión
}

\author{
Economic and social impact of personal assistance through the \\ methodology of Social Return on Investment
}

\begin{abstract}
Resumen
La figura del asistente personal es clave para que las personas con diversidad funcional puedan desarrollar sus proyectos de vida y hacer efectiva la vida independiente. A pesar de que la prestación de asistencia personal está recogida en el artículo 19 de la Ley 39/2006, de 14 de diciembre, de promoción de la autonomía personal y atención a las personas en situación de dependencia, ha sido poco promocionada y se presenta como un desafío en el avance por la igualdad de oportunidades. Este artículo muestra los resultados de una investigación que ha medido el impacto económico y social de un proyecto puesto en marcha por la Asociación Vida Independiente de Andalucía, en la que un grupo de siete personas con diversidad funcional han diseñado y ejecutado su propio proyecto de vida independiente. Se ha aplicado una metodología que calcula el Retorno Social de la Inversión, que se interesa no solo por los aspectos estrictamente económicos sino también sociales y ambientales. De los resultados obtenidos se desprenden los beneficios de la asistencia personal en la vida de las personas con diversidad funcional, sus familias y en las administraciones.
\end{abstract}

\section{Palabras clave}

Diversidad funcional, discapacidad, asistencia personal, vida independiente.

\author{
Antonio láñez Domínguez \\ <aiadom@upo.es> \\ Universidad Pablo de Olavide. \\ España
}

\section{José L. Aranda Chaves $<$ luisjosecha@gmail.com>}

Asociación de Enfermedades Neuromusculares de Andalucía (ASENSE-A). España

\section{Julia García Romero $<$ jgarciar@sevilla.org>}

Ayuntamiento de Sevilla. España

\begin{abstract}
The figure of personal assistant is key to people with functional diversity, as it helps them to develop their life projects and attain effectively independent lives. In Spain, Article 19 of Act 39/2006 of 14th December, on the development of personal autonomy and care for dependent people, includes the provision of personal assistance. Despite this, the act has been insufficiently implemented, making it challenging to advance equality of opportunities. Results are presented of a study that measured the economic and social impact of a project launched by the Independent Living Association of Andalusia, in which a group of seven people with functional diversity designed and executed their own Independent Life project. A methodology to calculate Social Return on Investment was used, which measures not only economic but also social and environmental aspects. Findings show the benefits that personal assistance brings to the lives of people with functional diversity, to their families and also to local authorities.
\end{abstract}

\section{Keywords}

Functional diversity, disability, personal assistance, independent living.

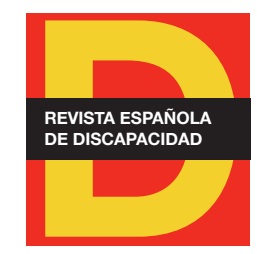

Para citar:

láñez, A. et al. (2018): "Impacto económico y social de la asistencia personal a través de la metodología del Retorno Social de la Inversión". Revista Española de Discapacidad, 6 (II): 81-102.

Doi: <https://doi.org/10.5569/23405104.06.02.05>

Fecha de recepción: 26-12-2017 Fecha de aceptación: 24-05-2018

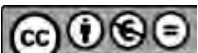


Introducción ${ }^{1}$

La asistencia personal es una prestación contemplada en el artículo 19 de la Ley 39/2006 de promoción de

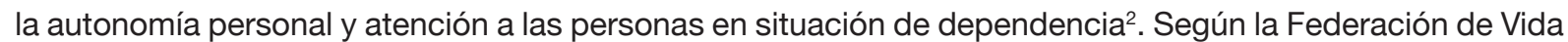
Independiente (FEVI), la asistencia personal es un servicio de apoyo a la vida independiente y la promoción de la autonomía de las personas con diversidad funcional. Mediante la figura del asistente personal permite a las personas con diversidad funcional asumir plena responsabilidad y control sobre sus vidas, proporciona soluciones personalizadas y posibilita así la independencia, la igualdad de oportunidades y la participación social ${ }^{3}$.

La asistencia personal emana del Movimiento de Vida Independiente, nacido en Estados Unidos a finales de los años 60 en el marco de la lucha por los derechos civiles. Fue impulsado por personas con diversidad funcional del ámbito universitario, con el objetivo de liberar a las personas de las instituciones cerradas, e incluso de sus propias casas, donde se encontraban recluidas, para integrarlas en la sociedad. Surge en el marco del paradigma social de la discapacidad, en su interés por fomentar la autonomía y la igualdad de oportunidades, y bajo la perspectiva de la filosofía de vida independiente, que parte de la idea de que "todas las personas tienen el derecho a la independencia a través del máximo control sobre sus vidas, basado en la capacidad y la oportunidad para tomar decisiones y de llevar a cabo las actividades de cada día" (láñez, 2010: 67). Para llevar a cabo esa independencia, la asistencia personal se convierte en un elemento de apoyo clave para que cada persona con diversidad funcional pueda desarrollar su proyecto de vida.

En nuestro país, esta prestación se ha incorporado de forma tardía con respecto a otros países europeos (Suecia, Noruega, Inglaterra o Irlanda), y cuando se materializa en la ley 39/2006, lo hace con un carácter restrictivo4; hecho que se evidencia con el escaso número de personas que son beneficiarias de la misma en comparación con aquellas que reciben otro tipo de prestaciones. $Y$ todo ello a pesar del reconocimiento "a vivir de forma independiente y a ser incluido en la comunidad" que recoge la Convención Internacional sobre los Derechos de las Personas con Discapacidad, en su artículo 19; un derecho basado en el principio fundamental de Derechos Humanos de que todos los seres humanos nacen iguales en dignidad y en derechos y que todas las vidas tienen el mismo valor.

El Movimiento de Vida Independiente en España ha realizado -y realiza- grandes esfuerzos para que la prestación de asistencia personal sea una realidad en nuestro país. En Andalucía, la asociación Vida Independiente Andalucía (en adelante VIAndalucía) viene trabajando para impulsar y desarrollar la asistencia personal en esta Comunidad Autónoma. Sus esfuerzos se recompensaron cuando el Parlamento de Andalucía aprobó por unanimidad una Proposición no de Ley (PNL) relativa a la asistencia personal para personas con

\footnotetext{
1. Este artículo está basado en el Informe publicado por láñez y Aranda en 2017, fruto de la investigación desarrollada para medir el impacto económico y social del proyecto de asistencia personal puesto en marcha por VIAndalucía, y estuvo financiada por la Universidad Internacional de Andalucía (UNIA).

2. Aunque la Ley $51 / 2003$, de 2 de diciembre, de igualdad de oportunidades, no discriminación y accesibilidad universal de las personas con discapacidad (LIONDAU) -actualmente derogada- ya hizo referencia en su artículo 9.1: "Las medidas de acción positiva podrán consistir en apoyos complementarios y normas, criterios y prácticas más favorables. Los apoyos complementarios podrán ser ayudas económicas, ayudas técnicas, asistencia personal, servicios especializados y ayudas y servicios auxiliares para la comunicación".

3. Véase: http://federacionvi.org/contenido/asistencia-personal/, acceso 19 de septiembre de 2016.

4. Se otorgará para el acceso a la educación y al trabajo, así como para una vida más autónoma en el ejercicio de las actividades básicas de la vida diaria (artículo 19).
} 
diversidad funcional ${ }^{5}$ en 2013. La PNL insta al Consejo de Gobierno a considerar la implantación y desarrollo de la prestación de asistencia personal y a posibilitar la puesta en marcha de un proyecto piloto hasta que se regule la prestación de forma efectiva y suficiente.

En el marco de la PNL, la Dirección General de Personas con Discapacidad de la Junta de Andalucía aprobó un proyecto piloto de asistencia personal a VIAndalucía, en 2014, a través de la convocatoria de subvenciones que esta Dirección General dispone para las entidades del sector de la diversidad funcional ${ }^{6}$. El proyecto se puso en marcha en marzo de 2015 y finalizó en agosto de 2016. Las personas beneficiarias fueron siete y ellas mismas -o sus familiares, en el caso de la diversidad funcional intelectual- han desarrollado su Plan Individual de Vida Independiente (PIVI), estimando el número de horas necesarias para desarrollar su proyecto de vida.

El carácter distintivo de este proyecto frente a otras iniciativas que se han puesto en marcha ha sido su acoplamiento con los principios que propugna la filosofía de vida independiente, entre otros la autoayuda (apoyo entre iguales), la posibilidad para ejercer poder (empoderamiento) y la responsabilidad sobre su propia vida y sus acciones. Las personas con diversidad funcional -o sus familiares- se convirtieron en gestoras del servicio, pues además de la selección de los asistentes personales, definieron en cada caso sus funciones, las actividades en las que participarían y los momentos en que estarían presentes a lo largo del día. Hasta entonces, en el análisis realizado sobre las iniciativas de vida independiente en España, señalaba Rueda (2013: 6): "Ninguno de los proyectos analizados se adapta plenamente al ideario de la Filosofía de Vida Independiente si bien su valor es importantísimo. Muestran la viabilidad y mayor eficacia de este tipo de políticas respecto de las tradicionales en cuanto a inversión económica, fuente de empleo y riqueza y, sobre todo, en lo que se refiere a hacer efectivos los derechos fundamentales y la igualdad de oportunidades de sus beneficiarios".

Esta nueva mirada a la hora de atender y hacer política hacia las personas con diversidad funcional motivó a realizar una investigación que midiera el impacto social y económico de la asistencia personal en el grupo de personas que iban a participar en el proyecto de VIAndalucía. Para ello, se aplicó la metodología del Retorno Social de la Inversión (Social Return on Investment, SROI por sus siglas en inglés) que calcula el retorno de la inversión, interesándose no sólo por los aspectos estrictamente económicos sino también sociales y ambientales.

Con este artículo queremos poner en valor la asistencia personal en tanto que los resultados que se desprenden de la investigación muestran los beneficios que ofrece este servicio, principalmente a las personas con diversidad funcional, sus familias y a las administraciones. Una evidencia que señaló Naciones Unidas (2014: 8) cuando indicaba que "la desinstitucionalización y el apoyo adecuado elevan la calidad de vida y mejoran las aptitudes funcionales individuales de las personas con discapacidad”.

5. BOPA Núm. 315 de 17 de octubre de 2013.

6. El proyecto piloto fue aprobado también en las Convocatorias de 2016 y 2017, aunque con una financiación menor. 


\section{La asistencia personal en España}

La ley 39/2006, de 14 de diciembre, de promoción de la autonomía personal y atención a las personas en situación de dependencia, define la asistencia personal como "el servicio prestado por un asistente personal que realiza o colabora en tareas de la vida cotidiana de una persona en situación de dependencia, de cara a fomentar su vida independiente, promoviendo y potenciando su autonomía personal" (artículo 2.7). Es por tanto un servicio que facilita la vida independiente a las personas con diversidad funcional, entendiendo por vivir independiente "que a las personas con discapacidades se les proporcionan todos los medios necesarios que les permitan ejercer la elección y el control sobre sus vidas y tomar las decisiones concernientes a sus vidas" (Naciones Unidas, 2017: 4). La figura del asistente personal es clave, ya que supone el apoyo necesario para desarrollar los proyectos de vida de las personas con diversidad funcional, hacer efectiva la vida independiente y lograr la igualdad de oportunidades.

Rodríguez-Picavea y Romañach (2006: 1) definen: “Un asistente personal es una persona que ayuda a otra a desarrollar su vida. El asistente personal es por tanto aquella persona que realiza o ayuda a realizar las tareas de la vida diaria a otra persona que por su situación, bien sea por una diversidad funcional o por otros motivos, no puede realizarlas por sí misma".

La asistencia personal es diferente a los cuidados familiares y a la ayuda a domicilio. Los cuidados familiares quedan circunscritos al ámbito familiar y se basan en la buena disposición de ambas partes. La ayuda a domicilio es un servicio profesionalizado, en manos normalmente de una empresa, en la que la persona con diversidad funcional no elige al trabajador y está orientado sobre todo a la realización de actividades de atención personal para la vida diaria y las actividades de ayuda doméstica (Urmeneta, 2011). Aunque el asistente personal puede realizar también estas funciones como parte de su trabajo, las suyas son variadas e incluso, en ocasiones, imprecisas. "Así pueden ser labores de acompañamiento, como realizar tareas tan específicas como pasar páginas, hacer la compra, conducir, tomar notas, marcar el teléfono, colocar cosas, limpiar, ayudar en la higiene personal, acompañar en el trabajo y un largo etcétera, que es imposible acotar" (Suñe y Martínez, 2015: 21).

Aunque esta figura ha sido desarrollada en otros países, en España, sigue siendo desconocida. La prestación de asistencia personal viene siendo una reivindicación constante por parte del Movimiento de Vida Independiente, si bien en los últimos años se está sumando el movimiento asociativo a esta nueva forma de entender la diversidad funcional. La Convención Internacional sobre los Derechos de las Personas con Discapacidad es clara a la hora de primar la autonomía personal y el derecho a vivir de forma independiente a lo largo de su articulado. Sin embargo, el número de personas beneficiarias es aún escaso, ya que es un servicio prestado desde algunas experiencias piloto que se han puesto en marcha por parte de ciertas comunidades autónomas, pero no se ha llegado a consolidar.

En el marco de la ley 39/2006, la representatividad de la asistencia personal, en comparación con el conjunto de prestaciones reconocidas en la ley, es de un 0,53\%. Ese porcentaje está marcado además por la particularidad del País Vasco, donde el volumen de personas beneficiaras es considerablemente superior que en el resto de comunidades autónomas, como se aprecia en la tabla 1, en la que se muestra el número de personas beneficiaras de las prestaciones recogidas en la ley, según el tipo de prestación que reciben. 


\begin{tabular}{|c|c|c|c|c|c|c|c|c|c|c|}
\hline \multirow[t]{2}{*}{$\begin{array}{l}\text { ÁMBITO } \\
\text { TERRITORIAL }\end{array}$} & 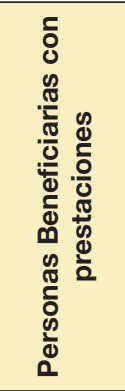 & 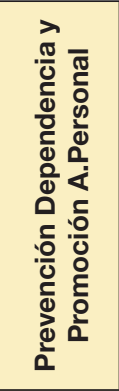 & 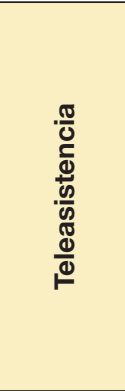 & 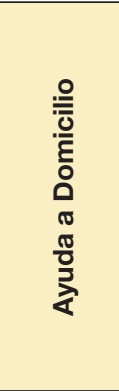 & 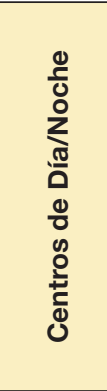 & 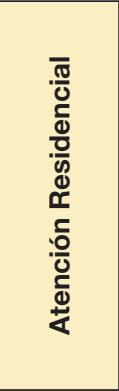 & 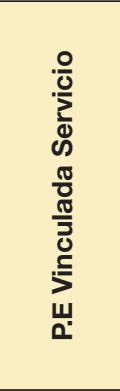 & 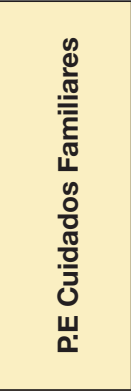 & 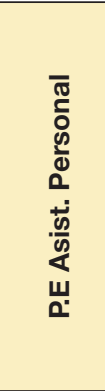 & TOTAL \\
\hline & $\mathbf{N}^{\circ}$ & $\mathbf{N}^{\circ}$ & $\mathbf{N}^{\circ}$ & $\mathbf{N}^{\circ}$ & $\mathbf{N}^{\circ}$ & $\mathbf{N}^{\circ}$ & $\mathbf{N}^{\circ}$ & $\mathbf{N}^{\circ}$ & $\mathbf{N}^{\circ}$ & $\mathbf{N}^{\circ}$ \\
\hline Andalucía & 196.489 & 1.587 & 80.329 & 61.989 & 13.885 & 24.012 & 3.600 & 69.936 & 9 & 255.347 \\
\hline Aragón & 26.705 & 3.204 & 1.161 & 2.995 & 1.530 & 3.864 & 5.378 & 12.133 & 0 & 30.265 \\
\hline $\begin{array}{l}\text { Asturias } \\
\text { (Principado de) }\end{array}$ & 21.575 & 6.194 & 1.061 & 3.972 & 2.326 & 3.175 & 2.462 & 8.466 & 1 & 27.657 \\
\hline Illes Balears & 18.113 & 3.011 & 2.537 & 690 & 1.125 & 2.468 & 818 & 12.096 & 0 & 22.745 \\
\hline Canarias & 19.953 & 17 & 840 & 8 & 4.375 & 3.733 & 3.859 & 7.953 & 0 & 20.785 \\
\hline Cantabria & 15.112 & 0 & 1.588 & 1.058 & 1.808 & 4.346 & 0 & 8.319 & 0 & 17.119 \\
\hline Castilla y León & 88.970 & 14.052 & 9.001 & 22.487 & 8.413 & 9.026 & 29.753 & 23.683 & 696 & 117.111 \\
\hline $\begin{array}{l}\text { Castilla- } \\
\text { La Mancha }\end{array}$ & 53.961 & 5.254 & 10.124 & 15.055 & 3.357 & 12.374 & 5.782 & 13.039 & 22 & 65.007 \\
\hline Catalunya & 148.069 & 912 & 18.921 & 27.948 & 12.364 & 29.634 & 13.196 & 81.152 & 19 & 184.146 \\
\hline $\begin{array}{l}\text { Comunitat } \\
\text { Valenciana }\end{array}$ & 67.202 & 601 & 3.474 & 52 & 6.641 & 11.206 & 11.185 & 37.202 & 6 & 70.367 \\
\hline Extremadura & 25.830 & 1.197 & 1.890 & 758 & 1.911 & 4.447 & 11.625 & 6.646 & 0 & 28.474 \\
\hline Galicia & 57.015 & 4.387 & 4.273 & 21.575 & 6.989 & 8.125 & 7.138 & 13.186 & 105 & 65.778 \\
\hline $\begin{array}{l}\text { Madrid } \\
\text { (Comunidad de) }\end{array}$ & 123.559 & 3.261 & 36.099 & 36.520 & 15.508 & 23.110 & 17.739 & 27.930 & 83 & 160.250 \\
\hline $\begin{array}{l}\text { Murcia } \\
\text { (Región de) }\end{array}$ & 33.916 & 3.679 & 5.731 & 670 & 3.623 & 4.278 & 1.499 & 22.789 & 0 & 42.269 \\
\hline $\begin{array}{l}\text { Navarra } \\
\text { (Comunidad } \\
\text { Foral de) }\end{array}$ & 12.655 & 603 & 2.176 & 1.060 & 299 & 1.827 & 1.104 & 9.373 & 3 & 16.445 \\
\hline País Vasco & 61.418 & 162 & 9.426 & 6.892 & 7.134 & 12.565 & 1.253 & 32.608 & 5.548 & 75.588 \\
\hline La Rioja & 7.948 & 686 & 2.377 & 2.700 & 888 & 1.396 & 1.080 & 2.007 & 0 & 11.134 \\
\hline Ceuta y Melilla & 2.694 & 427 & 618 & 829 & 84 & 218 & 5 & 1.413 & 0 & 3.594 \\
\hline TOTAL & 981.184 & 49.234 & 191.626 & 207.258 & 92.260 & 159.804 & 117.476 & 389.931 & 6.492 & 1.214 .081 \\
\hline \multicolumn{2}{|l|}{ PORCENTAJE } & $4,06 \%$ & $15,78 \%$ & $17,07 \%$ & $7,60 \%$ & $13,16 \%$ & $9,68 \%$ & $32,12 \%$ & $0,53 \%$ & $100,00 \%$ \\
\hline
\end{tabular}

Fuente: Imserso, situación a 31 de mayo de 2018.

En Andalucía, son nueve personas las que la reciben la prestación de asistencia personal, un porcentaje ínfimo en comparación con otras prestaciones, tales como la prestación por cuidados familiares, la ayuda a domicilio o los centros residenciales. 


\begin{tabular}{|c|c|c|}
\hline \multirow{2}{*}{$\begin{array}{l}\text { Prevención dependencia y } \\
\text { Promoción } \\
\text { A. Personal }\end{array}$} & $\mathbf{N}^{\circ}$ & $\%$ \\
\hline & 1.587 & 0,62 \\
\hline Teleasistencia & 80.329 & 31,46 \\
\hline Ayuda a Domicilio & 61.989 & 24,28 \\
\hline Centro de Día/Noche & 13.885 & 5,44 \\
\hline Atención Residencial & 24.012 & 9,40 \\
\hline P.E. Vinculada Servicio & 3.600 & 1,41 \\
\hline P.E Cuidados Familiares & 69.936 & 27,39 \\
\hline P.E. Asistencia Personal & 9 & 0,00 \\
\hline TOTAL & 255.347 & 100 \\
\hline
\end{tabular}

Fuente: Imserso, situación a 31 de mayo de 2018.

El desconocimiento de esta prestación entre las propias personas con diversidad funcional, entre los profesionales y entre la ciudadanía en general, la confusión con otras figuras como las de cuidador o auxiliar de ayuda a domicilio, la falta de facilidades por parte de la administración (por ejemplo, trabas administrativas a la hora de justificar los contratos y los pagos), la vinculación de la prestación al ámbito de la educación y el trabajo, así como la falta de voluntad política para darle un empuje a esta prestación, están detrás de esa escasa representatividad en el número de personas que la reciben (Urmeneta, 2011).

Si bien el asistente personal es una persona que realiza su trabajo de forma profesional y remunerada, no existe en el ordenamiento jurídico español una regulación de dicha figura, lo que dificulta poder formalizar la contratación laboral entre la parte y la contraparte de forma directa. Esta situación obliga a la persona con diversidad funcional a recurrir a empresas o entidades prestadoras de servicios o tendría que estar el asistente personal dado de alta como autónomo para prestar sus servicios. Por otro lado, es también una tarea pendiente definir la cualificación profesional del asistente personal.

\section{Características sociodemográficas de las personas participantes}

Las personas participantes del proyecto fueron siete. Si bien partimos de una muestra limitada en número, representa a su vez el universo, en cuanto que están contempladas todas las personas que se benefician de la asistencia personal a través del proyecto. Los resultados alcanzados están a su vez en consonancia con otras evaluaciones realizadas de proyectos similares.

Según el sexo, cinco fueron hombres y dos mujeres. Las edades estaban comprendidas entre los 16 y los 63 años, destacando que dos de las personas participantes eran menores de edad. 


\section{Gráfico 1. Edades de las personas participantes}

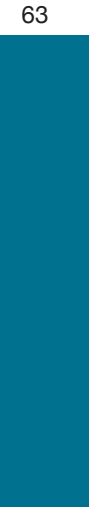

E1

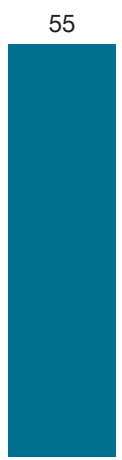

E2

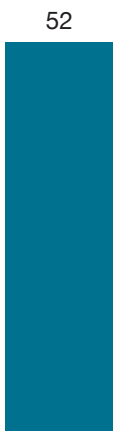

E3

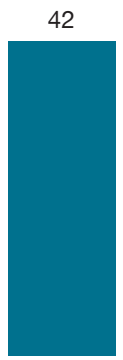

E4

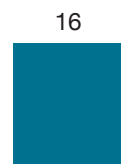

E5
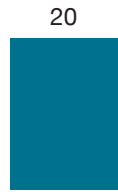

E6

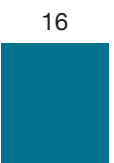

E7

Fuente: elaboración propia.

Según el tipo de diversidad funcional, 4 personas presentaban una diversidad funcional física (E1 a E4) y tres una diversidad funcional intelectual (E5 a E7). Los de menor edad eran las personas con diversidad funcional intelectual.

En cuanto al grado de discapacidad, todas las personas superaban el $65 \%$, con una oscilación entre el $67 \%$ y el $99 \%$. Según el grado de dependencia, cinco estaban en grado III, una persona en grado II y otra pendiente de valoración.

\begin{tabular}{l}
\hline Tabla 3. Grado de discapacidad de las personas participantes. Porcentajes \\
\begin{tabular}{|l|c|}
\hline Grado de discapacidad de las personas participantes & 88 \\
\hline E1 & 86 \\
\hline E2 & 99 \\
\hline E3 & 96 \\
\hline E4 & 77 \\
\hline E5 & 67 \\
\hline E6 & 69 \\
\hline E7 & 69 \\
\hline
\end{tabular}
\end{tabular}

Fuente: elaboración propia.

En cuanto al nivel formativo, las cuatro personas con diversidad funcional física tenían estudios universitarios y las tres personas con diversidad funcional intelectual tenían la categoría de estudiantes. Ninguna de ellas trabajaba de forma remunerada. 
Atendiendo a las prestaciones que recibían en el marco de la Ley 39/2006, tres personas recibían la prestación para cuidados en el entorno familiar y apoyo a cuidadores no profesionales, dos la prestación de asistencia personal, una el servicio de ayuda a domicilio y otra no recibía prestación debido a que estaba pendiente de ser valorada. En lo que refiere a la situación de convivencia, una persona vivía sola y seis en su entorno familiar.

El número de horas de asistencia personal fue determinado por las propias personas participantes en el proyecto, oscilando entre 14 y 84 horas semanales. Asimismo, cada participante seleccionó a sus asistentes personales y, en función de su organización personal y el número de horas, dispusieron de entre 1 y 3 asistentes personales para el desarrollo de su proyecto de vida independiente.

Tabla 4. Número de horas por semana y número de asistentes personales
\begin{tabular}{|l|c|c|}
\hline No horas de asistencia personal semanales & $\mathbf{N}^{\circ}$ de asistentes personales \\
\hline E1 & 14 & 2 \\
\hline E2 & 29 & 1 \\
\hline E3 & 84 & 3 \\
\hline E4 & 53,5 & 2 \\
\hline E5 & 38,5 & 2 \\
\hline E6 & 25 & 1 \\
\hline E7 & 38,5 & 1 \\
\hline
\end{tabular}

Fuente: elaboración propia.

De forma general, se puede constatar que el grupo de participantes en el proyecto presenta un perfil masculinizado, una media de edad cercana a los 38 años, con un elevado grado de diversidad funcional y con apoyos familiares. De forma más particular, si atendemos al tipo de diversidad funcional, se encuentran diferencias significativas. En el caso de la diversidad funcional intelectual, son las personas más jóvenes y están estudiando, mientras que las personas con diversidad funcional física están en la adultez y presentan un nivel de formación medio-alto. Las primeras requieren de apoyos por falta de autonomía moral y, en el caso de las segundas, por autonomía física.

\section{Metodología}

El SROI es una metodología que se inicia en San Francisco (California) en los años 90 y se desarrolla posteriormente en Inglaterra. Es una metodología basada en el principio de medición del valor extrafinanciero, es decir, toma en cuenta el valor económico, social y ambiental de una acción, proyecto o iniciativa, que no se refleja en la contabilidad financiera más tradicional. "Busca reducir la desigualdad y la degradación medioambiental, y mejorar el bienestar incorporando costos y beneficios sociales, medioambientales y económicos" (The SROI Network, 2013: 8). 
Entender el valor que se consigue con una inversión más allá de lo estrictamente económico está teniendo un interés cada vez mayor tanto en el sector público como privado, así como entre las entidades no lucrativas, por eso el SROI es una metodología útil no solo para las entidades financiadoras sino también para las entidades ejecutoras. "EI SROI mide el cambio en formas que son relevantes para las personas u organizaciones que lo experimentan o contribuyen con él. Cuenta cómo se genera el cambio midiendo los resultados (outcomes) sociales, medioambientales y económicos, y usa términos monetarios para representar dichos outcomes. Esto permite calcular el ratio costo-beneficio. Por ejemplo, un ratio 3:1 indica que una inversión de 1 genera 3 de valor social" (The SROI Network, 2013: 8).

Para aplicar esta metodología es necesario identificar e involucrar a los stakeholders (agentes de interés), que son todas aquellas personas y/u organizaciones que están implicadas en el proyecto y que, por tanto, van a experimentar cambios como consecuencia del desarrollo de la acción. Sus opiniones son importantes para llegar al objetivo y permiten que el analista vaya tomando decisiones. De igual modo, la identificación de los cambios es una tarea significativa, pues el SROI se basa en la medición de los cambios. Y todo ello con el fin último de valorar todo cambio, incluso aquellos que no están en el mercado financiero. Se lleva a cabo un proceso de monetización, asignando un valor monetario a cualquier cambio producido en los diferentes agentes de interés.

En sus inicios, esta metodología se aplicó para valorar los beneficios que generaban las inversiones realizadas en iniciativas empresariales orientadas a la creación de empleo de grupos en riesgo de exclusión social. En la actualidad, se utiliza para la evaluación de diferentes programas en los que se pretende medir el retorno social de una inversión. Es una metodología ventajosa porque permite medir y cuantificar el valor generado por una inversión, es decir, nos permite conocer el retorno social de cada euro invertido en una iniciativa o un proyecto. En España, en lo referido a la diversidad funcional, esta metodología se ha aplicado para medir el impacto de la asistencia personal en Barcelona (Ayuntamiento de Barcelona, 2012), en un estudio comparativo entre la asistencia personal y residencial en Madrid (Huete y Quezada, 2014), para el análisis del retorno social de la inversión pública en varios centros especiales de empleo en distintas comunidades autónomas (Ecodes, 2013, 2015a, 2015b), para el impacto del servicio canguro de Feaps-Aragón ${ }^{7}$ (Ecodes, 2014), etc.

El proceso de un análisis SROI sigue seis etapas ${ }^{8}$.

Gráfico 2. Fases de la Metodología SROI

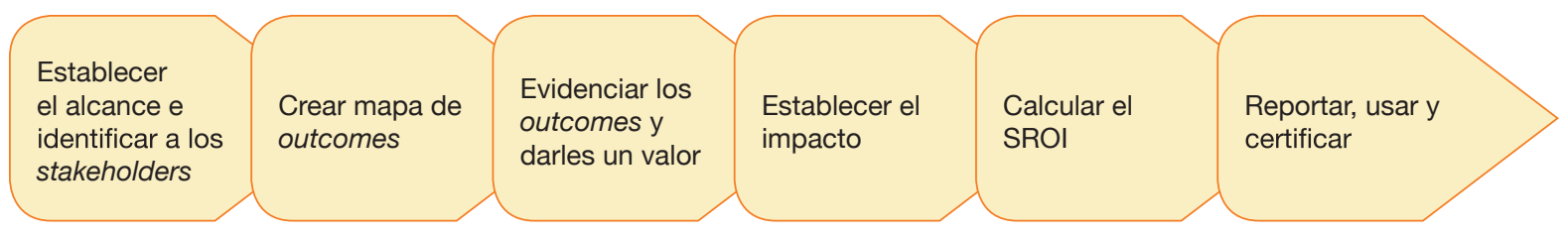

Fuente: elaboración propia.

7. Nota del editor: en la actualidad Plena inclusión Aragón.

8. Véase: http://observatoritercersector.org/canviepoca/wp-content/uploads/2014/02/OTS_Guide-SROI-spanhish.pdf. 
Desde el entendimiento de que la asistencia personal genera un valor no solo económico sino también social, nuestra investigación se llevó a cabo aplicando la metodología SROI. Los resultados se obtuvieron tras la aplicación de técnicas de investigación cuantitativas y cualitativas, incluyendo:

- Encuestas dirigidas a las personas usuarias del servicio de asistencia personal para conocer el perfil sociodemográfico.

- Análisis de los datos de carácter económico-financiero y de recursos humanos aportados por VIAndalucía.

- Análisis y valoración de referencias de otros estudios disponibles sobre el impacto y retorno de la inversión en programas para personas con diversidad funcional.

- Entrevistas a los distintos agentes de interés (personas con diversidad funcional, asistentes personales, entorno familiar, VIAndalucía y administraciones públicas) al objeto de identificar y valorar la importancia de los cambios percibidos durante la prestación del servicio.

- Contactos con los agentes de interés para contrastar la información recopilada.

\section{- Fase 1. Establecer el alcance e identificar a los stakeholders}

El primer paso de la metodología SROI es tener claro el alcance del análisis, es decir, qué se va a medir, e identificar los diferentes agentes de interés (stakeholders) implicados en la acción a desarrollar, especificando el papel que juega cada uno de ellos.

En nuestra investigación, el propósito del análisis ha sido medir el impacto social y económico del proyecto de asistencia personal que la Dirección General de Personas con Discapacidad de la Junta de Andalucía aprobó a VIAndalucía. El periodo de análisis comprendió desde marzo de 2015, momento de arranque del proyecto por parte de la asociación, hasta agosto de 2016 (17 meses). El grupo de stakeholders lo conformaron aquellas personas y/o entidades públicas y privadas que experimentarían cambios como consecuencia de la puesta en marcha del proyecto. Su identificación es clave en el análisis SROI, pues a través del diálogo establecido con ellos, evaluamos el valor que se genera en cada uno de los agentes de interés. En la tabla 5 se muestran los agentes y se ofrecen las razones para su participación en el análisis.

\begin{tabular}{|c|c|}
\hline Agentes de interés & Razón para su inclusión en el análisis \\
\hline $\begin{array}{l}\text { Administración Andaluza: } \\
\text { Dirección General de Personas con } \\
\text { Discapacidad }\end{array}$ & $\begin{array}{l}\text { - Responsable de la promoción de las políticas en materia de integración social de } \\
\text { las personas con diversidad funcional } \\
\text { - Organismo financiador principal del proyecto de asistencia personal a través de su } \\
\text { convocatoria pública }\end{array}$ \\
\hline Vida Independiente Andalucía & $\begin{array}{l}\text { - Responsable de la prestación del servicio y gestora del proyecto } \\
\text { - Encargada de contratar a los/as asistentes personales }\end{array}$ \\
\hline Asistentes personales & - Trabajadores que prestan el servicio \\
\hline $\begin{array}{l}\text { Personas con diversidad funcional } \\
\text { usuarias del servicio }\end{array}$ & - Principales beneficiarias directas del proyecto \\
\hline $\begin{array}{l}\text { Entorno familiar (cuidadores } \\
\text { principales) }\end{array}$ & $\begin{array}{l}\text { - Beneficiarias de la prestación, por la descarga de atención a su familiar con } \\
\text { diversidad funcional }\end{array}$ \\
\hline Administración General del Estado & $\begin{array}{l}\text { - Agente implicado indirectamente, ya que la contratación de los asistentes } \\
\text { personales le reporta beneficios por las cotizaciones a la Seguridad Social, por la } \\
\text { recaudación del IRPF y por el ahorro en las prestaciones por desempleo }\end{array}$ \\
\hline
\end{tabular}

Fuente: elaboración propia. 
Se hace necesario señalar que estos agentes fueron los principales involucrados en el proyecto y los que obtuvieron el mayor beneficio, pero el efecto e impacto que tiene este tipo de acción repercute también en otros agentes, tales como los amigos, los vecinos así como en la sociedad en general. Sin embargo, no se tuvieron en cuenta por estar fuera de los límites de la investigación.

\section{- $\quad$ Fase 2. Crear el mapa de outcomes}

De cada agente de interés se debe especificar la inversión realizada (inputs), que pueden ser contribuciones económicas, recursos humanos, tiempo, etc. La suma de estos recursos supondría el coste total de la acción. Asimismo, se describen las actividades (outputs) de forma cuantitativa, que pueden llevarse a cabo con esa inversión, y se identifican los cambios (outcomes) resultantes de las actividades desarrolladas. El mapa muestra la relación entre inputs, outputs y outcomes.

La construcción de nuestro mapa de impactos se realizó atendiendo a la participación de cada uno de los stakeholders considerados como principales, poniendo en relación los recursos económicos y humanos invertidos, las actividades llevadas a cabo y los resultados identificados para cada stakeholder. Esto es lo que se denomina cadena de valor del impacto.

Tabla 6. La cadena de valor del impacto

\begin{tabular}{|c|c|c|c|c|}
\hline \multicolumn{2}{|c|}{ Plan de Trabajo del Proyecto Social } & \multicolumn{2}{c|}{ Resultados previstos del Proyecto Social } \\
\hline 1. Insumos & 2. Actividades & 3. Productos & 4. Resultados & 5. Impactos \\
\hline $\begin{array}{c}\text { Recursos } \\
\text { humanos y de } \\
\text { capital invertidos } \\
\text { en la actividad }\end{array}$ & $\begin{array}{c}\text { Actuaciones concretas del } \\
\text { proyecto }\end{array}$ & $\begin{array}{c}\text { Productos } \\
\text { tangibles } \\
\text { derivados de la } \\
\text { actividad }\end{array}$ & $\begin{array}{c}\text { Resultados teniendo en } \\
\text { cuenta lo que pudo haber } \\
\text { actividad } \\
\text { sucedido sin la actividad, } \\
\text { la acción de otros y los } \\
\text { resultados sobrevenidos }\end{array}$ \\
\hline
\end{tabular}

Fuente: Hehenberger et al. (2013).

Los inputs/insumos refieren a todos los recursos que se aportan y son necesarios para la puesta en marcha del proyecto. En cuanto a lo económico, por un lado lo conformó la inversión realizada por la Dirección General de Personas con Discapacidad a través de su convocatoria pública (150.570 €), y por otro, las contribuciones de aquellas personas beneficiarias del proyecto, que participaron en la financiación del servicio con el importe de la prestación que perciben a través de la Ley 39/2006 (35.673,93 €). En lo referente a los recursos humanos, se tuvo en cuenta el tiempo dedicado a la gestión del proyecto por VIAndalucía (15.782,60 €), realizado sobre una estimación de 20 horas a la semana de acuerdo con la información analizada y conforme a la ganancia media por hora trabajada en Andalucía. La inversión económica total ha ascendido a $202.026,53 €$. 


\begin{tabular}{l}
\hline Tabla 7. Identificación de los inputs \\
\begin{tabular}{|l|c|}
\hline Identificación de los inputs & $150.570,00 €$ \\
\hline Dirección General de Personas con Discapacidad & $35.673,93 €$ \\
\hline Prestaciones de las personas beneficiarias & $15.782,60 €$ \\
\hline Recursos humanos de VIAndalucía & $\mathbf{2 0 2 . 0 2 6 , 5 3 €}$ \\
\hline TOTAL & \\
\hline
\end{tabular}
\end{tabular}

Fuente: elaboración propia.

Los outputs corresponden a las diferentes actividades que se han llevado a cabo fruto de la inversión ejecutada. En nuestro caso, concierne a la prestación del servicio de asistencia personal a las siete personas con diversidad funcional, la contratación de los doce asistentes personales y las 18.788,50 horas dedicadas a la prestación del servicio durante los 17 meses de duración del proyecto.

Tabla 8. Descripción de los outputs

\begin{tabular}{|c|}
\hline Descripción de los outputs \\
\hline Servicio de asistencia a personal a siete personas con diversidad funcional \\
\hline Contratación de doce asistentes personales \\
\hline $18.788,50$ horas de servicio prestado \\
\hline
\end{tabular}

Fuente: elaboración propia.

Los outcomes son los resultados alcanzados con la puesta en marcha del servicio de asistencia personal. Para recoger la información de los outcomes se aplicó la técnica de la entrevista semiestructurada en profundidad y se recurrió al registro de la información que VIAndalucía llevaba a cabo para la justificación de la subvención concedida. Los cambios se identificaron entrevistando a la persona con diversidad funcional, a un familiar-cuidador y también a un asistente personal de cada caso. Las entrevistas se llevaron a cabo entre los meses de diciembre de 2015 a marzo de 2016, lo que significó que en ese momento las personas llevaban con el servicio de asistencia personal entre diez y doce meses.

Dado que la Dirección General de Personas con Discapacidad y VI-Andalucía formaban parte de los agentes de interés, también se han realizado entrevistas a informantes clave de estas entidades.

Todas las entrevistas se registraron y transcribieron para su posterior análisis. Se utilizó el programa informático atlas.ti como herramienta de análisis de datos. Para el análisis cuantitativo y la aplicación del SROI se generaron plantillas en el programa Excel. 


\begin{tabular}{|c|c|c|}
\hline $\begin{array}{l}\text { Agentes de } \\
\text { interés }\end{array}$ & Medibles & No medibles \\
\hline $\begin{array}{l}\text { Administración } \\
\text { Andaluza: } \\
\text { Dirección General } \\
\text { de Personas con } \\
\text { Discapacidad }\end{array}$ & $\begin{array}{l}\text { - Ahorro por la reducción del uso de otras } \\
\text { prestaciones sociales }\end{array}$ & $\begin{array}{l}\text { - Reducción del consumo de servicios } \\
\text { - Panitarios } \\
\text { Política más cercana a la Convención } \\
\text { Internacional sobre los Derechos de las } \\
\text { Personas con Discapacidad } \\
\text { - Cumplimiento -en parte- de la } \\
\text { Proposición No de Ley, aprobada en el } \\
\text { Parlamento de Andalucía }\end{array}$ \\
\hline $\begin{array}{l}\text { Vida Independiente } \\
\text { Andalucía } \\
\end{array}$ & $\begin{array}{l}\text { - Incremento de recursos humanos voluntarios } \\
\text { - Mayor visibilidad de la asociación }\end{array}$ & $\begin{array}{l}\text { - Avance en su objetivo de promover la } \\
\text { asistencia personal y sensibilización social }\end{array}$ \\
\hline $\begin{array}{l}\text { Asistentes } \\
\text { personales }\end{array}$ & $\begin{array}{l}\text { - Mayores conocimientos relacionados con la atención } \\
\text { - Obersonal } \\
\text { - Motivación de un saliario por su trabajo } \\
\text { - Mejora de las sinergias entre los asistentes } \\
\text { personales y usuarios del servicio }\end{array}$ & $\begin{array}{l}\text { - Visibilidad de la figura del asistente } \\
\text { personal } \\
\text { - Aumento de demanda para trabajar como } \\
\text { asistente personal }\end{array}$ \\
\hline $\begin{array}{l}\text { Personas con } \\
\text { diversidad } \\
\text { funcional usuarias } \\
\text { del servicio }\end{array}$ & 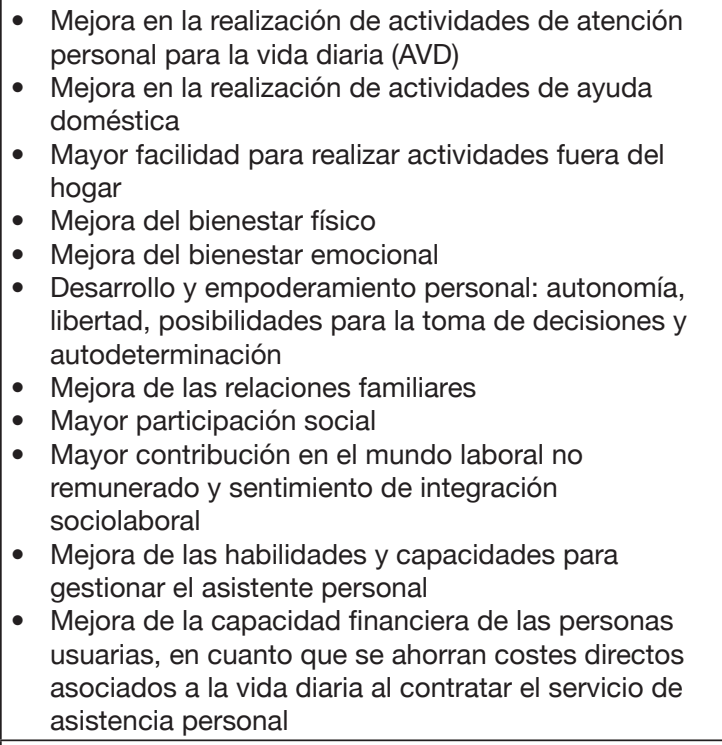 & $\begin{array}{l}\text { - Inquietud e incertidumbre a tomar } \\
\text { decisiones sobre planes a medio y largo } \\
\text { plazo, dada la temporalidad del proyecto }\end{array}$ \\
\hline $\begin{array}{l}\text { Entorno familiar } \\
\text { (cuidadores/as } \\
\text { principales) }\end{array}$ & $\begin{array}{l}\text { - Mejora del bienestar emocional } \\
\text { - Recuperación y mejora de sus relaciones sociales } \\
\text { - Reducción del número de horas de dedicación y } \\
\text { apoyo a su familiar con diversidad funcional } \\
\text { Mayores oportunidades laborales gracias a la } \\
\text { descarga de atención del familiar principal }\end{array}$ & $\begin{array}{l}\text { - Dificultad de integrar al asistente personal } \\
\text { en la vida familiar }\end{array}$ \\
\hline $\begin{array}{l}\text { Administración } \\
\text { General del Estado }\end{array}$ & $\begin{array}{l}\text { - Incremento de ingresos de fondos vía cotizaciones } \\
\text { de la Seguridad Social de los trabajadores } \\
\text { - Incremento de ingresos de fondos vía cotizaciones } \\
\text { de la Seguridad Social empresarial } \\
\text { - Incremento del ingreso de fondos vía recaudación } \\
\text { del IRPF } \\
\text { - Ahorro en prestaciones por desempleo }\end{array}$ & \\
\hline
\end{tabular}

Fuente: elaboración propia. 


\section{- Fase 3. Evidenciar los outcomes y darles un valor}

Identificados los outcomes, se definieron los indicadores que permitían determinar el grado de cumplimiento de los resultados obtenidos y posteriormente asignarles un valor monetario. Para determinar si los outcomes contemplados en la etapa anterior han ocurrido y en qué medida, se analizó y evaluó en profundidad la información recopilada de las entrevistas realizadas a los distintos agentes implicados y se determinó un indicador por cada cambio contemplado.

En el análisis SROI, a pesar de la dificultad para medir ciertos cambios, se le da valor a todo resultado, de aquí la diferencia con las evaluaciones más tradicionales en las que sólo se tienen en cuenta aquellos resultados susceptibles de valoración económica. Ese proceso de valoración se conoce como monetización y se calcula sobre aproximaciones (proxies) o valores sustitutivos, al ser imposible obtener una medida exacta por no tener un valor monetario en el mercado. En el conjunto de los cambios, hay algunos que fueron más fácilmente cuantificables y objetivables y otros fueron más relativos y subjetivables. Estos últimos fueron sometidos a consideración por parte de las personas usuarias del servicio de asistencia personal. Se les envió el mapa de outcomes para que cada persona, desde su subjetividad, estableciera los proxies más oportunos con que medir los cambios dados en sus vidas desde la puesta en marcha del servicio.

\section{- Fase 4. Establecer el impacto}

Antes de calcular el SROI, es preciso reflexionar sobre qué cambios han sucedido gracias a la acción puesta en marcha y cuáles no, de modo que, para ser justos, no se pueden atribuir cambios que habrían sucedido aun no habiéndose desarrollado la acción (peso muerto) o se han logrado debido a otros factores (atribución). En estos casos, los cambios no serían imputados a la acción, sino que quedarían fuera de consideración. Es por ello que establecer el impacto fue indispensable para valorar los efectos del servicio de asistencia personal, pues se trataba de determinar en qué medida los cambios se debían a la puesta en marcha del proyecto. Se evaluaron las estimaciones y se ajustaron los cálculos de los impactos teniendo en cuenta otros factores, personas y organizaciones que pudieran influir. Para ello, se aplicaron los correctores de peso muerto (deadweight) y atribución en función del impacto medido.

\begin{tabular}{|c|c|c|c|}
\hline $\begin{array}{l}\text { Agentes de } \\
\text { interés }\end{array}$ & Aproximación financiera/Financial proxy & Valor & Fuente \\
\hline $\begin{array}{l}\text { Administración } \\
\text { Andaluza: } \\
\text { Dirección General } \\
\text { de personas con } \\
\text { discapacidad }\end{array}$ & $\begin{array}{l}\text { Ahorro medio por usuario del servicio de } \\
\text { asistencia personal en otras prestaciones }\end{array}$ & $7.134,79 €$ & Cuestionario usuarios y la Ley 39/2006 \\
\hline \multirow{2}{*}{$\begin{array}{l}\text { Vida } \\
\text { Independiente } \\
\text { Andalucía }\end{array}$} & $\begin{array}{l}\text { Salario mínimo interprofesional de } \\
\text { trabajadores eventuales, temporeros y } \\
\text { empleados del hogar }\end{array}$ & $5,13 €$ & Real Decreto $1171 / 2015$ de 29 de diciembre \\
\hline & $\begin{array}{l}\text { Coste de una campaña publicitaria en radio } \\
\text { y televisión de Andalucía }\end{array}$ & $4.444,00 €$ & Canal Sur Radio y Televisión \\
\hline
\end{tabular}




\begin{tabular}{|c|c|c|c|}
\hline $\begin{array}{l}\text { Agentes de } \\
\text { interés }\end{array}$ & Aproximación financiera/Financial proxy & Valor & Fuente \\
\hline \multirow{4}{*}{$\begin{array}{l}\text { Asistentes } \\
\text { personales }\end{array}$} & $\begin{array}{l}\text { Curso de formación sobre asistencia } \\
\text { personal }\end{array}$ & $328,80 €$ & $\begin{array}{l}\text { Coste medio de cursos sobre asistencia } \\
\text { personal }\end{array}$ \\
\hline & $\begin{array}{l}\text { Salario medio percibido según convenio } \\
\text { (descontando S. Social e IRPF) }\end{array}$ & $10.540,76 €$ & $\begin{array}{l}\text { VIAndalucía y XIV Convenio colectivo } \\
\text { general de centros y servicios de atención a } \\
\text { personas con discapacidad }\end{array}$ \\
\hline & Curso de motivación y satisfacción laboral & $140,00 €$ & $\begin{array}{l}\text { Coste medio de cursos sobre motivación de } \\
\text { personal }\end{array}$ \\
\hline & $\begin{array}{l}\text { Curso de habilidades de comunicación y } \\
\text { conductas en PDF }\end{array}$ & $226,67 €$ & $\begin{array}{l}\text { Coste medio de cursos sobre habilidades } \\
\text { de comunicación y destrezas }\end{array}$ \\
\hline \multirow{11}{*}{$\begin{array}{l}\text { Personas con } \\
\text { diversidad } \\
\text { funcional usuarias } \\
\text { del servicio }\end{array}$} & $\begin{array}{l}\text { Coste/hora asistente personal en el mercado } \\
\text { privado }\end{array}$ & $12,39 €$ & $\begin{array}{l}\text { Coste medio de varias empresas de servicio } \\
\text { de asistencia personal en Andalucía }\end{array}$ \\
\hline & Coste/hora empleados del hogar & $10,20 €$ & $\begin{array}{l}\text { Coste medio de varias empresas de servicio } \\
\text { de limpieza en Andalucía }\end{array}$ \\
\hline & $\begin{array}{l}\text { Coste/hora asistente personal en el mercado } \\
\text { privado }\end{array}$ & $12,39 €$ & $\begin{array}{l}\text { Coste medio de varias empresas de servicio } \\
\text { de asistencia personal en Andalucía }\end{array}$ \\
\hline & Coste tratamiento de fisioterapia & $5.110,00 €$ & $\begin{array}{l}\text { Coste medio de diferentes consultas de } \\
\text { fisioterapia }\end{array}$ \\
\hline & Coste tratamiento psicológico individual & $1.445,00 €$ & $\begin{array}{l}\text { Tarifa media de diferentes consultas de } \\
\text { psicología }\end{array}$ \\
\hline & Coste una silla ruedas alta gama & $8.624,74 €$ & $\begin{array}{l}\text { Coste medio de una silla de ruedas } \\
\text { eléctrica alta gama }\end{array}$ \\
\hline & Coste de una terapia psicológica familiar & $1.657,50 €$ & $\begin{array}{l}\text { Tarifa media de diferentes consultas de } \\
\text { psicología }\end{array}$ \\
\hline & Cuota de socio a un club social & $1.036,31 €$ & Coste medio de diferentes clubs sociales \\
\hline & $\begin{array}{l}\text { Salario mínimo interprofesional de } \\
\text { trabajadores/as eventuales, temporeros/as y } \\
\text { empleados/as del hogar }\end{array}$ & $5,13 €$ & Real Decreto $1171 / 2015$ de 29 de diciembre \\
\hline & Curso de gestión y desarrollo personal & $416,67 €$ & $\begin{array}{l}\text { Coste medio de varios cursos de gestión y } \\
\text { desarrollo personal }\end{array}$ \\
\hline & $\begin{array}{l}\text { Ahorro medio experimentado por cada } \\
\text { usuario/a durante el periodo }\end{array}$ & $4.431,99 €$ & $\begin{array}{l}\text { Entrevistas a usuarios/as y evaluación } \\
\text { durante el periodo }\end{array}$ \\
\hline \multirow{4}{*}{$\begin{array}{l}\text { Entorno familiar } \\
\text { (Cuidadores } \\
\text { principales) }\end{array}$} & Coste tratamiento psicológico individual & $1.445,00 €$ & $\begin{array}{l}\text { Tarifa media de diferentes consultas de } \\
\text { psicología }\end{array}$ \\
\hline & Cuota de socio a un club social & $1.036,31 €$ & Coste medio de diferentes clubs sociales \\
\hline & $\begin{array}{l}\text { Coste/hora personal en los servicios de } \\
\text { atención a la dependencia }\end{array}$ & $10,05 €$ & $\begin{array}{l}\text { VI Convenio colectivo estatal de servicios } \\
\text { de atención a personas dependientes y } \\
\text { desarrollo de promoción a la autonomía } \\
\text { personal }\end{array}$ \\
\hline & $\begin{array}{l}\text { Ganancia media por hora trabajada en } \\
\text { Andalucía }\end{array}$ & $10,81 €$ & Instituto Nacional de Estadística \\
\hline \multirow{4}{*}{$\begin{array}{l}\text { Administración } \\
\text { General del } \\
\text { Estado }\end{array}$} & $\begin{array}{l}\text { Cotización Seguridad Social practicada a } \\
\text { los trabajadores }\end{array}$ & $8.569,63 €$ & VIAndalucía \\
\hline & Cotización Seguridad Social de la empresa & $43.589,51 €$ & VIAndalucía \\
\hline & $\begin{array}{l}\text { Retenciones del IRPF practicadas a los } \\
\text { trabajadores }\end{array}$ & $2.612,91 €$ & VIAndalucía \\
\hline & Prestación media por desempleo percibida & $13.600,68 €$ & Ministerio de Trabajo y Seguridad Social \\
\hline
\end{tabular}

Fuente: elaboración propia. 


\section{- Fase 5. Calcular el SROI}

Esta etapa consiste en sumar todos los impactos positivos, restando aquellos negativos y comparando este resultado con los recursos invertidos, obteniendo así el coeficiente SROI. Por cada euro invertido en el servicio de asistencia personal para personas con diversidad funcional física e intelectual se ha conseguido retornar a la sociedad -en valor socio-económico- $3,62 €$.

En la tabla 11 se detalla la valoración sobre el retorno social del servicio de asistencia personal, desglosado por los grupos de interés considerados como relevantes en esta investigación.

\begin{tabular}{|c|c|c|c|}
\hline INVERSIÓN TOTAL & IMPORTE INVERSIÓN & \multirow{2}{*}{$\begin{array}{l}\text { Por cada } 1 € \\
\text { invertido }\end{array}$} & \multirow{2}{*}{$\begin{array}{l}\text { DISTRIBUCIÓN DEL } \\
\text { IMPACTO TOTAL }\end{array}$} \\
\hline APORTACIONES PÚBLICAS & $186.243,93 €$ & & \\
\hline $\begin{array}{l}\text { Subvención de la Dirección General de Personas con } \\
\text { Discapacidad destinada a la prestación del Servicio } \\
\text { de Asistencia Personal }\end{array}$ & $150.570,00 €$ & & \\
\hline $\begin{array}{l}\text { Ayudas percibidas por las personas usuarias de } \\
\text { la asistencia personal a través de Ley 39/2006 y } \\
\text { destinadas al servicio }\end{array}$ & $35.673,93 €$ & & \\
\hline APORTACIONES PRIVADAS & $15.782,60 €$ & & \\
\hline $\begin{array}{l}\text { Recursos humanos destinados por VIAndalucía a la } \\
\text { prestación del servicio }\end{array}$ & $15.782,60 €$ & & \\
\hline INVERSIÓN TOTAL & $202.026,53 €$ & & \\
\hline AGENTES DE INTERÉS (STAKEHOLDERS) & TOTAL IMPACTO & $\begin{array}{l}\text { IMPACTO POR } \\
\text { AGENTE }\end{array}$ & $\%$ POR AGENTE \\
\hline Administración Autonómica. Junta de Andalucía & $35.673,94 €$ & $0,18 €$ & $4,88 \%$ \\
\hline VIAndalucía & $11.489,40 €$ & $0,06 €$ & $1,57 \%$ \\
\hline Asistentes personales & $40.598,52 €$ & $0,20 €$ & $5,55 \%$ \\
\hline $\begin{array}{l}\text { Personas con diversidad funcional usuarias del } \\
\text { servicio de asistencia personal }\end{array}$ & $319.785,31 €$ & $1,58 €$ & $43,74 \%$ \\
\hline Entorno familiar/cuidador & $219.767,58 €$ & $1,09 €$ & $30,06 \%$ \\
\hline Administración General del Estado & $103.754,50 €$ & $0,51 €$ & $14,19 \%$ \\
\hline $\begin{array}{l}\text { IMPACTO ECONÓMICO Y SOCIAL TOTAL QUE } \\
\text { RETORNA A LA SOCIEDAD }\end{array}$ & $731.069,25 €$ & $3,62 €$ & $100,00 \%$ \\
\hline RATIO DE RETORNO & $3,62 €$ & & \\
\hline
\end{tabular}

Fuente: elaboración propia.

\section{- Fase 6. Reportar, usar y certificar}

Es conveniente socializar los resultados con los stakeholders para recoger sus opiniones y sugerencias. Con toda la información procesada, se elaboró un informe preliminar con los resultados, que se envió a las personas con diversidad funcional -o familiares, dependiendo del tipo de diversidad funcional- para que pudieran aportar cualquier sugerencia y/o elemento que lo enriqueciese. Ha sido un proceso participativo 
en el que la opinión e implicación de las personas beneficiaras ha sido fundamental para la aplicación de la metodología SROI. El análisis SROI finalizó con la entrega del informe a los stakeholders, albergando la esperanza de que pueda servirle a las administraciones públicas a la hora de diseñar sus políticas sociales.

\section{Conclusiones}

La investigación ha evidenciado los cambios que ha generado el proyecto de asistencia personal en cada uno de los agentes de interés. Todos esos cambios se han cuantificado, siguiendo la metodología SROI, y se ha logrado obtener una aproximación al valor -en términos monetarios- que ha creado el servicio de asistencia personal. Los resultados obtenidos inducen a poner en valor la asistencia personal, debido al retorno social que produce: por cada $1 €$ invertido se retornan 3,62 €. El valor monetario de todos los impactos económicos y sociales del servicio de asistencia personal de VIAndalucía sobre la inversión total realizada $(202.026,53 €)$ ha alcanzado la cifra de 731.049,25 €.

\section{Gráfico 3. Distribución del valor total por agente de interés}

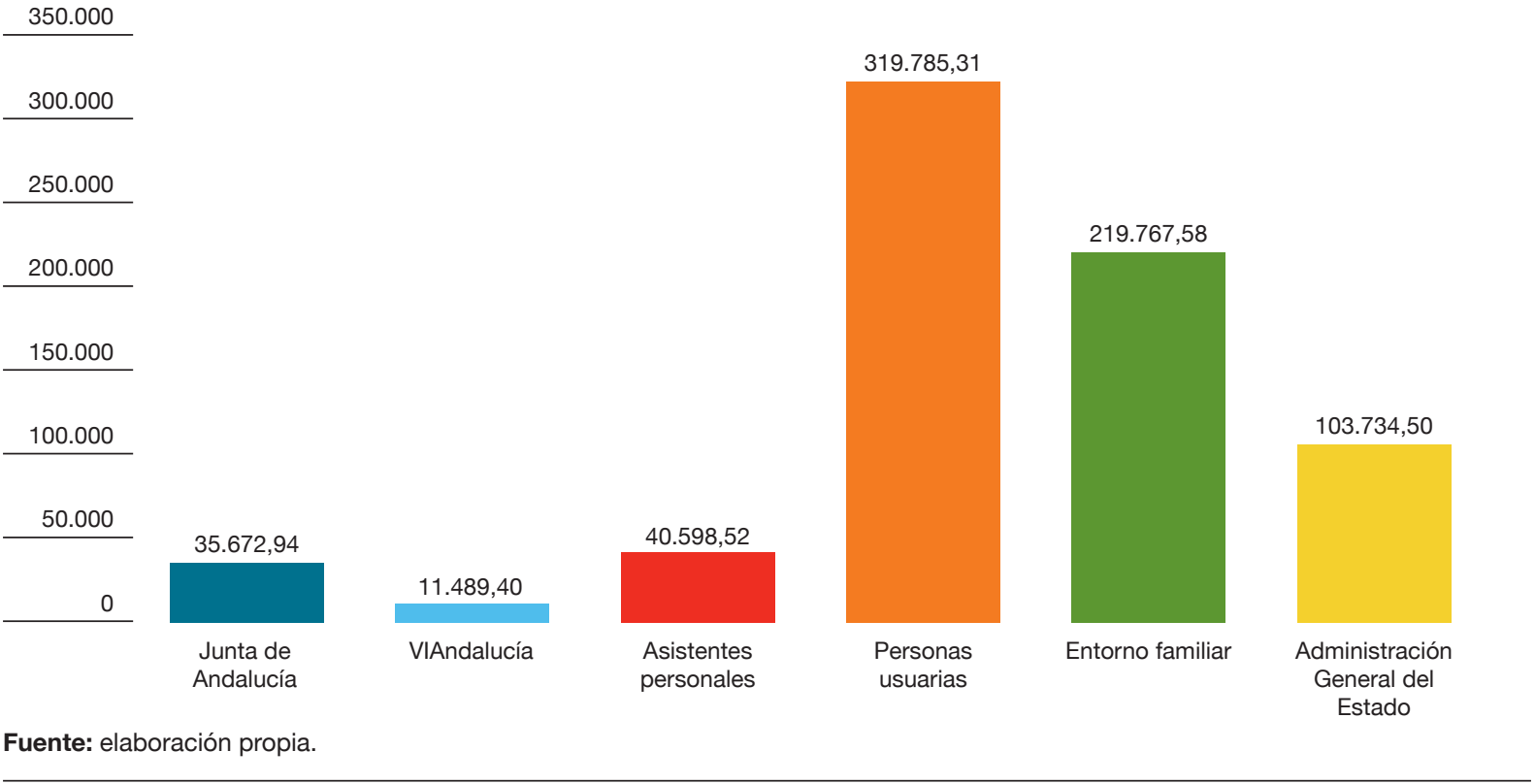

El mayor impacto recae en las personas con diversidad funcional, al ser el agente que recibe directamente el servicio de asistencia personal, ascendiendo a 319.785,31€, un 43,74\% del retorno total. En la línea de los principios que emana de la filosofía de vida independiente, estas personas consiguieron un mayor desarrollo personal y empoderamiento, pues vieron incrementada su autonomía, su libertad, sus posibilidades para la toma de decisiones y su autodeterminación. 


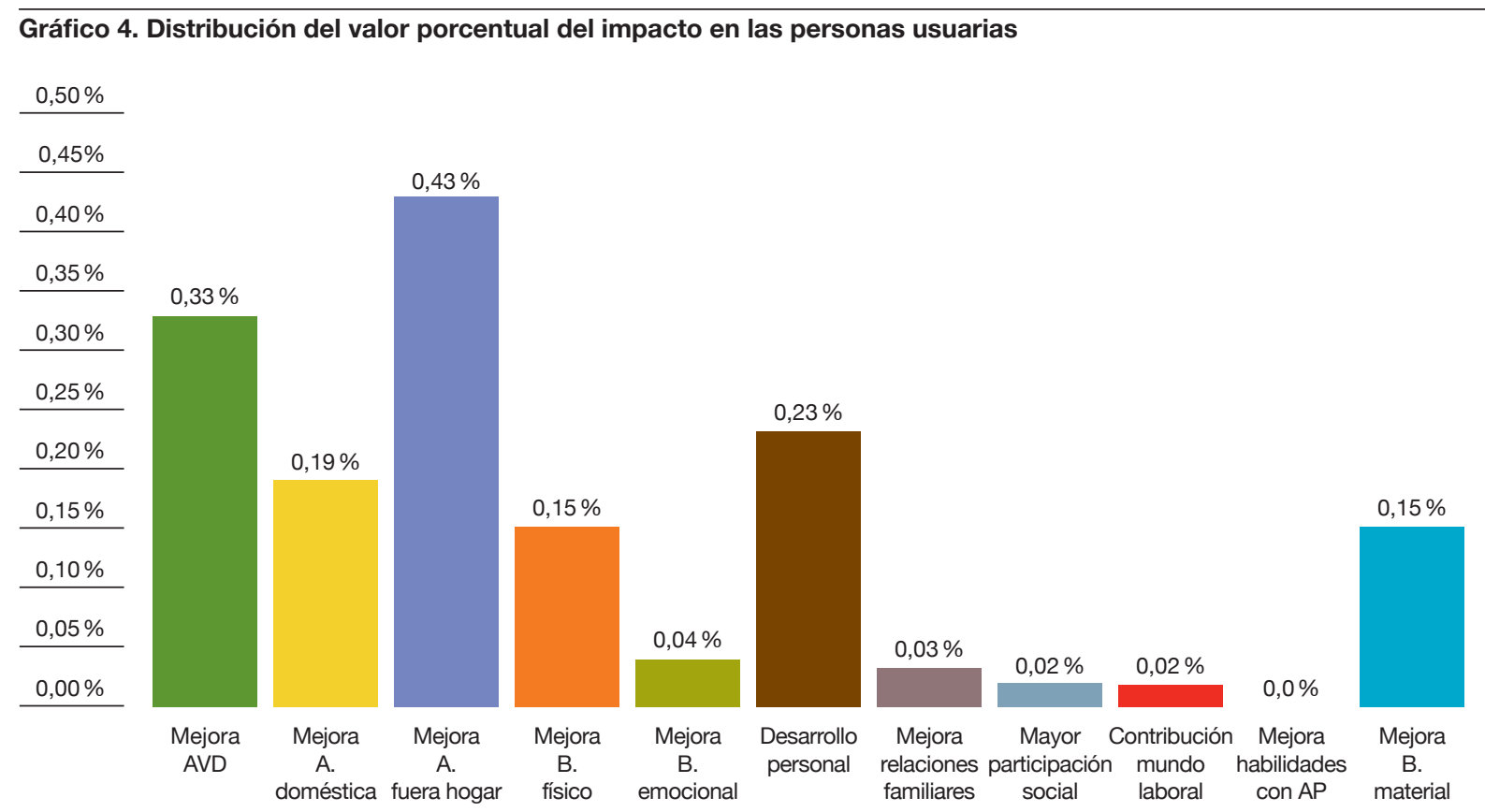

Fuente: elaboración propia.

Los cambios más significativos los representan la mejora en la realización de las actividades fuera del hogar y en las actividades de atención personal para la vida diaria. Las personas con diversidad funcional han sentido que sus vidas se han dignificado, ya que los apoyos prestados de forma profesional por los asistentes personales les daban el control para tomar libremente sus decisiones, mientras que los apoyos familiares les hacen sentirse, en ocasiones, atrapadas en una situación de dependencia al estar sujeta la relación a la buena disposición de ambas partes. De depender de los favores de sus familiares y/o amistades han pasado a tener la tranquilidad y la seguridad de que un profesional prestará los apoyos para ser independientes.

De igual modo, sintieron cambios en su entorno, señalando la mejora de las relaciones familiares y su mayor participación social. En la medida que se sentían más independientes, su poder de participación era mayor. La asistencia personal posibilitó también un aumento de la contribución al mundo laboral no remunerado y el sentimiento de integración sociolaboral.

El proyecto de asistencia personal ha permitido un ahorro potencial a las personas beneficiarias si hubiesen tenido que contratarlo en el mercado privado. Claro está que las circunstancias socioeconómicas de estas personas no permitirían la contratación del servicio de forma privada en las mismas condiciones en las que lo han estado recibiendo. Por consiguiente, se hace necesaria la financiación pública de este servicio para que todas las personas con diversidad funcional tengan las mismas oportunidades para alcanzar la vida independiente, sin que el estatus económico sea motivo de desigualdad.

Se identificó un cambio negativo asociado a la temporalidad del proyecto (sujeto a la convocatoria de subvenciones de la Dirección General de Personas con Discapacidad). La inquietud e incertidumbre a la hora de 
tomar decisiones sobre planes a medio y largo plazo condicionó la implementación de acciones por parte de las personas beneficiarias.

El entorno familiar es el agente que se sitúa en segunda posición en el cúmulo de impactos, un 30,06\% del impacto total, concentrándose el mayor porcentaje en la mejora del bienestar material, ya que se produce una reducción del tiempo de dedicación a los cuidados proporcional al número de horas de asistencia personal que recibe la persona con diversidad funcional. La recuperación de horas les ofrece una mejora del bienestar emocional, más tiempo libre para su ocio y mayores oportunidades para la búsqueda de empleo, no obstante este último ha tenido menor influencia en el análisis dada la avanzada edad de los cuidadores principales, pero consideramos importante señalar la relevancia de este impacto. El total de impactos para el entorno familiar ha sido de 219.767,58 €. También en el entorno familiar se reconoció un impacto negativo en lo referido a la incorporación del asistente personal en la vida familiar, por la pérdida de intimidad que supone esa figura externa. No obstante, todos los casos manifestaron que era una cuestión de adaptación, sobrevalorando las ventajas y beneficios de la asistencia personal frente a esta dificultad.

La Administración General del Estado recibe un retorno económico directo de 54.772,05 €, mediante los ingresos procedentes de las cotizaciones a la Seguridad Social y la recaudación del Impuesto sobre la Renta de las Personas Físicas (IRPF). Por otra parte, recibe ahorros indirectos por valor de 48.962,45 € derivados de la reducción de prestaciones por desempleo como consecuencia de la contratación de los asistentes personales. La suma de estos impactos asciende al 14,19\% del retorno total y supone, de manera indirecta, una disminución considerable de la inversión pública neta destinada al servicio de asistencia personal.

En el caso de los asistentes personales, se produce un claro impacto directo procedente de los salarios que reciben por su trabajo, que ascendieron a 126.489,14 €. Además, hay que destacar otros impactos, como la adquisición de conocimientos y habilidades en el desempeño de su trabajo, que perduran más allá del periodo de análisis. Todos ellos manifestaron durante las entrevistas su motivación y satisfacción por las tareas que desempeñaban. Los impactos valorados suman 40.598,52 €, un 5,5\% del retorno total.

El impacto generado en la Administración Andaluza, valorado en 35.673,94 €, proviene del ahorro en el gasto por la reducción de las prestaciones sociales que perciben las personas usuarias en el marco de la Ley $39 / 2006$. Este impacto supone $4,88 \%$ del retorno total. Asimismo, la aprobación del proyecto supuso un paso importante en el cumplimiento -en parte- de la Proposición No de Ley, aprobada en el Parlamento de Andalucía, que sirve de base para impulsar la asistencia personal. También se comprobó una reducción en el consumo de servicios sanitarios, pero resultó poco significativo en el periodo analizado y, por tanto, no se ha cuantificado.

Por su parte, VIAndalucía ha recibido impactos valorados en 11.489,40 €, un 1,57 \% del impacto, gracias al trabajo voluntario de sus miembros y al aumento de la visibilidad como asociación en la esfera pública y privada. También, como entidad gestora del servicio, ha conseguido otros impactos que no se han cuantificado, como la promoción de la autonomía personal y la sensibilización de la sociedad y otras organizaciones del sector sobre la figura del asistente personal.

Además de estos impactos, se ha podido constatar que la asistencia personal es una prestación solicitada y demandada por un determinado perfil. Las personas participantes contaban con una serie de condicio- 
nes favorecedoras para recibir el servicio de asistencia personal: red de apoyo familiar, nivel de formación medio-alto (bien sea por ellas mismas o por sus familiares, en el caso de la diversidad funcional intelectual) y capacidades para gestionar la asistencia personal. En la medida que avanzaba el servicio, las personas con diversidad funcional -o sus familiares- vieron mejoradas sus habilidades y capacidades para gestionar la asistencia personal.

La modalidad de 'autogestión' en la que se ha llevado a cabo el proyecto ha exigido que las personas contasen con una serie de habilidades y capacidades para tomar decisiones y saber dirigir su propio proyecto personal. El modo de poner en marcha esta prestación 'autogestionada' ha permitido una mayor rentabilidad en el número de horas dedicadas al servicio, pues al no haber intermediación pública ni privada de por medio, no han existido costes de personal.

La incipiente puesta en marcha de la asistencia personal en España y el poco desarrollo que ha tenido aún, requieren de una mayor promoción para que se conozca realmente el sentido práctico de la misma, a la vez que una regularización de esta figura en la que se le asigne una categoría profesional y establezca un marco laboral. La asistencia personal supone una fuente de creación de empleo, por tanto, su regulación y su reconocimiento profesional permitirían una mejora de sus condiciones laborales.

La Convención Internacional sobre los Derechos de las Personas con Discapacidad ha significado un cambio importante en la manera de entender la 'discapacidad'. El nuevo enfoque que plantea, bajo el prisma del modelo social, debe plasmarse en las políticas sociales, que deben promover servicios adecuados y variados para que cada persona con diversidad funcional decida y elija lo que más le interesa para sí misma. 
Ayuntamiento de Barcelona (2012): Evaluación del impacto social y del servicio de asistente personal del Instituto Municipal de Personas con Discapacidad (en línea). <http://estatic.bcn.cat/BarcelonaAccessible/Continguts/ Documents/Impacto_Social_Servicio_Assistente_Personal_CAST.pdf>, acceso 6 de noviembre de 2015.

Ecodes (2015a): Medición del retorno económico y social de las entidades adheridas a FEAFES mediante la aplicación de la metodología SROI (en línea). <https://www.cedd.net/es/buscar/Record/530646>, acceso 31 de octubre de 2015.

Ecodes (2015b): Análisis del retorno social de la inversión en Laboratorio DAU mediante la aplicación de la metodología SROI (en línea). <http://fundaciodau.barcelona.ppe.entitats.diba.cat/wp-content/uploads/sites/76/2016/04/Informe_SROI_DAU_CET_12.2015.pdf>, acceso 3 de octubre de 2015.

Ecodes (2014): Medición del impacto económico y social de los servicios sociales para las personas con discapacidad intelectual y sus familias en Aragón mediante la aplicación de la metodología SROI (en línea). <https:// ecodes.org/component/option,com_phocadownload/Itemid,2/download,289/id,134/view,category/>, acceso 2 de diciembre de 2015.

Ecodes (2013): Análisis del retorno social de la inversión pública en un CEE de iniciativa social mediante la aplicación de la metodología SROI (en línea). <http://ecodes.org/phocadownload/Informe_SROI_CEE_2013.pdf>, acceso 7 de noviembre de 2015.

España. Ley 39/2006, de 14 de diciembre, de promoción de la autonomía personal y atención a las personas en situación de dependencia, Boletín Oficial del Estado, 15 de diciembre de 2006, núm. 299, pp. 44142-44156.

España. Ley 51/2003, de 2 de diciembre, de igualdad de oportunidades, no discriminación y accesibilidad universal de las personas con discapacidad, Boletín Oficial del Estado, 3 de diciembre de 2003, núm. 289, pp. 43187-43195.

Hehenberger, L. et al. (2013): A Practical Guide to Measuring and Managing Impact (en línea). <http://www.oltreventure.com/wp-content/uploads/2015/05/EVPA_A_Practical_Guide_to_Measuring_and_-Managing_Impact_ final.pdf >, acceso 1 de octubre de 2015.

Huete, A. y Quezada, M. Y. (2014): Análisis econométrico. Estudio comparativo de la asistencia personal y la atención residencial (en línea). <http://www.madrid.org/bvirtual/BVCM013850.pdf>, acceso 7 de noviembre de 2015.

láñez, A. y Aranda, J. L. (2017). Impacto económico y social del proyecto de asistencia personal de VIAndalucía (en línea). <https://www.siis.net/documentos/ficha/519694.pdf>, acceso 2 de mayo de 2018.

láñez, A. (2010): Prisioneros del cuerpo. La construcción social de la diversidad funcional. A Coruña: Diversitas.

Imserso (2018): Información Estadística del Sistema para la Autonomía y Atención a la Dependencia (en línea). <http://www.dependencia.imserso.es/InterPresent1/groups/imserso/documents/binario/estsisaad20180531. pdf>, acceso 6 de julio de 2018.

Naciones Unidas (2017). Living independently and being included in the community (en línea). <http://tbinternet. ohchr.org/_layouts/treatybodyexternal/TBSearch.aspx?Lang=en\&TreatyID=4\&DocTypelD=11>, acceso 2 de mayo de 2018. 
Naciones Unidas (2014): Thematic study on the right of persons with disabilities to live independently and be included in the community. Report of the Office of the United Nations High Commissioner for Human Rights (en línea). <http://www.asksource.info/resources/thematic-study-right-persons-disabilities-live-independentlyand-be-included-community>, acceso 2 de mayo de 2018.

Naciones Unidas (2006): Convención Internacional sobre los Derechos de las Personas con Discapacidad. Nueva York: ONU.

Rodríguez-Picavea, A. y Romañach, J. (2006): Consideraciones sobre la figura del asistente personal en el proyecto de ley de promoción de la autonomía personal y atención a las personas en situación de dependencia (en línea). <http://www.asoc-ies.org/vidaindepen/docs/la_\%20figura_del_asistente_personal_v1-1.pdf>, acceso 5 de junio de 2016.

Rueda, M. (2013): Análisis comparativo de las iniciativas de vida independiente en España (en línea). <http://forovidaindependiente.org/analisis-comparativo-de-las-iniciativas-de-vida-independiente-en-espana/>, acceso 9 de junio de 2016.

Suñe, A. y Martínez, I. (2015): “La figura del asistente personal”, en Ortega, E. (coord.): Situación de la asistencia personal en España. Madrid: Predif.

The SROI Network (2013): A Guide to Social Return on Investment. The Cabinet Office. Reino Unido. Traducido y adaptado al español por el Grupo CIVIS. <http://observatoritercersector.org/canviepoca/wp-content/ uploads/2014/02/OTS_Guide-SROI-spanhish.pdf>, acceso 6 de septiembre de 2015.

Urmeneta, X. (2011): Vida independiente y asistencia personal. La experiencia de Gipuzkoa (en línea). <http://forovidaindependiente.org/vida_independiente_y_asistencia_personal/>, acceso 2 de junio de 2016. 